\title{
MicroRNA-652 promotes cell proliferation and osteosarcoma invasion by directly targeting KLF9
}

\author{
YONGPING JIN $^{1}$, LIU YANG ${ }^{2}$ and XIA LI ${ }^{1}$ \\ ${ }^{1}$ Institute of Nursing Allied Health Sciences, College of Nursing and Health Science, Henan University; \\ ${ }^{2}$ Department of Nursing, Kaifeng Health School, Kaifeng, Henan 475001, P.R. China
}

Received August 7, 2018; Accepted August 12, 2019

DOI: $10.3892 /$ etm.2020.9037

\begin{abstract}
Previous studies have demonstrated that various microRNAs (miRNAs or miRs) are abnormally expressed in osteosarcoma(OS) and serve roles in its malignant development. An in-depth understanding of the specific roles of dysregulated miRNAs in OS may be important for cancer research and the identification of novel therapeutic targets. In the current study, reverse transcription-quantitative PCR was performed to determine miR-652 expression in OS tissues and cell lines. Cell Counting Kit- 8 and Transwell invasion assays were used for assessing the effect of miR-652 on the proliferation and invasion of OS cells. Herein, miR-652 expression was assessed in OS and the effects and molecular mechanisms of miR-652 in OS cells were examined. The results revealed that miR-652 expression was significantly upregulated in OS tissues and cell lines compared with adjacent normal tissues and a normal human osteoblast cell line. Furthermore, miR-652 downregulation inhibited the proliferation and invasion of OS cells. miR-652 was also demonstrated to directly interact with the 3 '-untranslated region of kruppel-like factor 9 (KLF9) and miR-652 negatively regulated KLF9 expression in OS cells. miR-652 and KLF9 mRNA levels were also revealed to be inversely correlated in OS tissues. Treatment with KLF9 small interfering RNA abolished the suppression of OS proliferation and invasion induced by miR-652 downregulation. miR-652 may serve an oncogenic role in OS cells by targeting KLF9 directly. The results also indicated that miR-652 may be an effective novel therapeutic target for the treatment of patients with OS.
\end{abstract}

Correspondence to: Professor Xia Li, Institute of Nursing Allied Health Sciences, College of Nursing and Health Science, Henan University, North Section of Jinming Road, Kaifeng, Henan 475001, P.R. China

E-mail: lixia_henan@163.com

Key words: microRNA-652, osteosarcoma, kruppel-like factor 9, proliferation, invasion

\section{Introduction}

Osteosarcoma (OS) is derived from primitive bone mesenchymal cells and is the most common malignant bone tumor (1). OS primarily occurs in children and young adults between 10-20 years of age and is characterized by lung metastasis and a poor prognosis, posing a serious threat to health (2). Multiple factors, including epigenetic alterations, environmental ionizing radiation and lesions, have been demonstrated to be associated with OS formation and progression (3). However, the complex molecular mechanisms associated with OS development remain unclear. Currently, surgical resection in combination with preoperative and postoperative chemotherapy is a standard treatment for patients with OS (4). Despite years of development in OS diagnosis and treatment, therapeutic outcomes have not improved (5). Therefore, an understanding of OS pathogenesis is required so that more specific therapeutic targets can be identified for patients with this fatal malignant tumor.

Recent studies have demonstrated that microRNAs (miRNAs or miRs) serve crucial roles in different types of human cancer (6-8). miRNAs are a series of non-coding, short RNA molecules that negatively regulate gene expression via sequence-specific interactions with the 3'-untranslated regions (UTR) of their target gene (9). These interactions suppress translation and/or induce the degradation of mRNAs (10). The dysregulation of miRNAs has been demonstrated in nearly all types of human malignancy, including bladder cancer (11), colorectal cancer (12), lung cancer (13) and cervical cancer (14). A variety of miRNAs are differentially expressed in OS and their deregulation is involved in OS genesis and development $(15,16)$. miRNAs serve oncogenic or tumor suppressive roles, and participate in the regulation of a variety of pathological processes, including cell proliferation, the cell cycle, apoptosis, metastasis and epithelial-mesenchymal transition (17-19). Identifying the roles of dysregulated miRNAs in OS may contribute to the development of techniques for improved diagnosis, therapy and prognosis.

miR-652 is an miRNA that has been studied in pancreatic cancer (20) and non-small cell lung cancer (21). However, the specific role and underlying mechanism of miR-652 in OS has not yet been elucidated. In the current study, the expression of miR-652 was examined in OS tissues and cell lines. The effects of miR-652 on the biological role of OS cells were 
examined, and the molecular mechanisms underlying the activity of miR-652 in OS cells were also assessed.

\section{Materials and methods}

Tumor specimen collection. A total of 29 pairs of OS and adjacent normal tissues ( $2 \mathrm{~cm}$ away from tumor tissues) were obtained from 29 patients (age range, 12-27 years; 17 males, 12 females) between May 2015 and March 2017 from The First Affiliated Hospital of Henan University (Kaifeng, China). No patients were subjected to chemotherapy, radiotherapy or other treatments prior to surgical resection and none were suffering from any other disease. All tissues were snap-frozen in liquid nitrogen and stored at $-80^{\circ} \mathrm{C}$. The current study was approved by the Ethics Committee of Henan University. Written informed consent was provided by all participants or their legal guardians prior to enrollment in the current study.

Cell lines. A total of three human OS cell lines (U2OS, MG-63 and HOS) and a normal human osteoblast (hFOB1.19) were purchased from the Type Culture Collection of the Chinese Academy of Sciences. All cell lines were cultured in DMEM supplemented with $10 \%$ FBS and $1 \%$ penicillin/streptomycin mixture (all, Gibco; Thermo Fisher Scientific, Inc.), and grown at $37^{\circ} \mathrm{C}$ in a humidified incubator supplied with $5 \% \mathrm{CO}_{2}$.

Oligonucleotides, small interfering RNA (siRNA) and cell transfection. miR-652 mimics, miRNA mimic negative controls (miR-NC), miR-652 inhibitors, negative control (NC) inhibitors, siRNA against the expression of kruppel-like factor 9 (KLF9) and NC siRNA were all produced by Shanghai GenePharma Co., Ltd. U2OS and HOS cells were plated into six-well plates at a density of $6 \times 10^{5}$ cells/well 1 day prior to transfection at $37^{\circ} \mathrm{C}$. The following sequences were used: miR-652 mimics, 5'AAUGGCGCCACUAGGGUUGUG3'; miR-NC, 5'UUCUCCGAACGUGUCACGUTT3'; miR-652 inhibitor, 5'UUACCGCGGUGAUCCCAACAC3'; NC inhibitor, 5'CAG UACUUUUGUGUAGUACAA3'; KLF9 siRNA, 5'CAGUUC CGCUGUCCGCUGU3' and NC siRNA, 5'UUCUCCGAA CGUGUCACGUTT3'. Cells were subsequently transfected transiently with 100 pmol miR-652 mimic, 100 pmol miR-NC, 100 pmol miR-652 inhibitor, 100 pmol NC inhibitor, 100 pmol KLF9 siRNA or 100 pmol NC siRNA using Lipofectamine 2000 (cat. no. 11668019; Invitrogen; Thermo Fisher Scientific, Inc.), according to manufacturer's protocol. FBS-free DMEM was used during transfection. At $6 \mathrm{~h}$ after transfection, culture medium was replaced with DMEM containing 10\% FBS. Reverse transcription-quantitative (RT-q)PCR and a transwell invasion assay were performed at $48 \mathrm{~h}$ post-transfection. A Cell Counting Kit- 8 (CCK-8) assay and western blotting were performed at 24 and $72 \mathrm{~h}$ after transfection.

Reverse transcription-quantitative PCR (RT- $q P C R)$. RT-qPCR analysis was performed to detect miR-652 and KLF9 mRNA expression form OS tissues, adjacent normal tissues, OS cell lines (U2OS, MG-63 and HOS) and a normal human osteoblast (hFOB1.19). The TRIzol ${ }^{\circledR}$ reagent (Invitrogen; Thermo Fisher Scientific, Inc.) was used to isolate total RNA from tissue specimens or cells following the manufacturer's protocol. To measure mRNA expression, total RNA was converted to
cDNA using a TaqMan miRNA Reverse Transcription kit and subjected to qPCR which was performed using a TaqMan miRNA PCR kit (all, Applied Biosystems; Thermo Fisher Scientific, Inc.). The temperature protocol for RT was as follows: $16^{\circ} \mathrm{C}$ for $30 \mathrm{~min}, 42^{\circ} \mathrm{C}$ for $30 \mathrm{~min}$ and $85^{\circ} \mathrm{C}$ for $5 \mathrm{~min}$. The thermocycling conditions for PCR were: $50^{\circ} \mathrm{C}$ for $2 \mathrm{~min}$; $95^{\circ} \mathrm{C}$ for $10 \mathrm{~min} ; 40$ cycles of denaturation at $95^{\circ} \mathrm{C}$ for $15 \mathrm{sec}$ and annealing/extension at $60^{\circ} \mathrm{C}$ for $60 \mathrm{sec}$. To quantify KLF9 mRNA expression, cDNA was prepared from total RNA using a PrimeScript RT Reagent kit (Takara Bio, Inc.). qPCR was subsequently performed using a SYBR Premix Ex Taq ${ }^{\mathrm{TM}}$ II kit (Takara Bio, Inc.). The temperature protocol for RT was: $37^{\circ} \mathrm{C}$ for $15 \mathrm{~min}$ and $85^{\circ} \mathrm{C}$ for $5 \mathrm{sec}$. qPCR was then performed with the following thermocycling conditions: $5 \mathrm{~min}$ at $95^{\circ} \mathrm{C}$; 40 cycles of $95^{\circ} \mathrm{C}$ for $30 \mathrm{sec}$ and $65^{\circ} \mathrm{C}$ for $45 \mathrm{sec}$. miR- 652 expression was normalized to that of U6 small nuclear RNA and KLF9 mRNA was normalized to that of GAPDH. Relative gene expression was quantified using the $2^{-\Delta \Delta C q}$ method (22). The primer sequences were as follows: miR-652 forward, 5'-ACACTCCAGCTGGGCAACCCTAGGAGAGGGTGC-3' and reverse, 5'-GTGTCGTGGAGTCGGCAATTC-3'; U6 forward, 5'-GCTTCGGCAGCACATATACTAAAAT-3' and reverse, 5'-CGCTTCACGAATTTGCGTGTCAT-3'; KLF9 forward, 5'-ACAGTGGCTGTGGGAAAGTC-3' and reverse, 5'-TCACAAAGCGTTGGCCAGCG-3'; GAPDH forward 5'-TGGTATCGTGGAAGGACTC-3' and reverse, 5'-AGTAGA GGCAGGGATGATG-3'.

CCK-8 assay. Following transfection, U2OS and HOS cells were incubated for $24 \mathrm{~h}$ at $37^{\circ} \mathrm{C}$ in a humidified incubator containing $5 \% \mathrm{CO}_{2}$. Cells were collected and subsequently seeded into 96-well plates with an initial density of $2 \times 10^{3}$ cells/well. After incubations at $37^{\circ} \mathrm{C}$ for $0,24,48$ and $72 \mathrm{~h}$, a CCK- 8 assay (Dojindo Molecular Technologies, Inc.) was performed to determine cell proliferation according to the manufacturer's protocol. A total of $10 \mu \mathrm{l}$ CCK-8 solution was added into each well prior to incubation at $37^{\circ} \mathrm{C}$ for an additional $2 \mathrm{~h}$. Absorbance was detected at a wavelength of $450 \mathrm{~nm}$ using an Enzyme Immunoassay Analyzer (Bio-Rad Laboratories, Inc.).

Transwell invasion assay. Transfected U2OS and HOS cells were incubated at $37^{\circ} \mathrm{C}$ with $5 \% \mathrm{CO}_{2}$ for $48 \mathrm{~h}$, harvested and resuspended in FBS-free DMEM. In total, $5 \times 10^{4}$ transfected cells suspended in FBS-free DMEM medium were plated into the upper compartments of a transwell chamber that were precoated with Matrigel (all, BD Biosciences). The precoating was conducted in a $37^{\circ} \mathrm{C}$ incubator for $4-5 \mathrm{~h}$. The lower compartments were coated with $500 \mu 1$ DMEM containing $20 \%$ FBS. After $24 \mathrm{~h}$ of incubation at $37^{\circ} \mathrm{C}$, cells that had invaded through the membrane were fixed with $4 \%$ paraformaldehyde at $37^{\circ} \mathrm{C}$ for $20 \mathrm{~min}$ and stained with $0.05 \%$ crystal violet at $37^{\circ} \mathrm{C}$ for $30 \mathrm{~min}$. Non-invading cells were scraped off using a cotton swab. The number of invading cells was counted with a CKX41 inverted light microscope (magnification x200; Olympus Corporation).

Bioinformatics analysis. The putative targets of miR-652 were predicted using TargetScan Human 7.1 (http://www. targetscan.org/vert_71/) and microRNA.org (http://www. microrna.org/microrna/). 
Luciferase reporter assay. The 3'-UTR regions of the human KLF9 gene containing the putative wild-type or mutant miR-652 binding sequences were amplified by Shanghai GenePharma Co., Ltd., cloned into a pGL3 luciferase vector (Promega Corporation) and respectively named pGL3-KLF9-3'-UTR wild-type and pGL3-KLF9-3'-UTR mutant. U2OS and HOS cells were inoculated into 24-well plates at a density of $1.5 \times 10^{5}$ cells/well and after $24 \mathrm{~h}$ incubation at $37^{\circ} \mathrm{C}$, the recombined luciferase reporter plasmids and miR-652 inhibitor or NC inhibitor were transfected into cells using Lipofectamine $2000^{\circledR}$. At $48 \mathrm{~h}$ after transfection, luciferase activity was determined using a dual-luciferase Reporter Assay system (Promega Corporation). Firefly luciferase activities were normalized to that of Renilla luciferase activity.

Western blot analysis. After washing twice with cold PBS, total protein was extracted from transfected U2OS and HOS cells using RIPA lysis buffer (Upstate Biotechnology, Inc.). Total protein was subsequently detected using a BCA assay kit (Beyotime Institute of Biotechnology). Equal quantities of protein $(30 \mu \mathrm{g} /$ lane) were loaded for SDS-PAGE on $10 \%$ polyacrylamide gels and transferred to PVDF membranes (EMD Millipore). After blocking with 5\% fat-free milk at room temperature for $2 \mathrm{~h}$, the membranes were washed three times with Tris-buffered saline containing $0.1 \%$ Tween-20 and incubated overnight at $4^{\circ} \mathrm{C}$ with the following primary antibodies: Rabbit anti-human KLF9 (1:1,000; cat. no. ab227920; Abcam) and rabbit anti-human GAPDH (1:1,000; cat. no. ab128915; Abcam). GAPDH was used as an internal reference to confirm equal protein loading. Membranes were then further probed with horseradish peroxidase-conjugated goat anti-rabbit IgG secondary antibodies (1:5,000; cat. no. ab6721; Abcam) at room temperature for $2 \mathrm{~h}$. Signals were visualized using an enhanced chemiluminescence detection system (Pierce; Thermo Fisher Scientific, Inc.), and were analyzed with Quantity One software version 4.62 (BioRad Laboratories, Inc.).

Statistical analysis. All data were presented as the mean \pm standard deviation. SPSS software version 17.0 (SPSS Inc.) was used to perform statistical analysis. A paired Student's t-test (for parametric data) or a Wilcoxon signed-rank test (for non-parametric data) was used to analyze data between two groups, while a one-way ANOVA followed by a Tukey's post hoc test was used to compare the differences between three or more groups. The association between miR-652 and KLF9 mRNA levels in OS tissue was assessed using Spearman's correlation analysis. $\mathrm{P}<0.05$ was considered to indicate a statistically significant result.

\section{Results}

miR-652 is highly expressed in human OS tissues and cell lines. To reveal the expression pattern of miR-652 in OS, RT-qPCR analysis was performed using 29 pairs of OS tissue and adjacent normal tissue. The results revealed that miR-652 expression was significantly higher in OS tissues compared with adjacent normal tissues (Fig. 1A; $\mathrm{P}<0.05$ ). The expression of miR-652 in three human OS cell lines (U2OS, MG-63 and HOS) and a normal human osteoblast cell line (hFOB1.19) was also assessed. The results demonstrated that miR-652 expression was significantly higher in all three OS cell lines compared with hFOB1.19 cells (Fig. 1B; $\mathrm{P}<0.05$ ). The results indicated that the upregulation of miR-652 may serve an important role in the pathogenesis of OS.

miR-652 inhibition impairs OS cell proliferation and invasion. To determine the functional roles of miR-652 in OS, U2OS and HOS cells were transfected with a miR-652 inhibitor or $\mathrm{NC}$ inhibitor. These cell lines were selected as they exhibited a relatively higher miR-652 expression than the other cell lines assessed. RT-qPCR analysis revealed that miR-652 expression was significantly downregulated in U2OS and HOS cells after transfection with the miR-652 inhibitor (Fig. 2A; $\mathrm{P}<0.05$ ). The regulatory effect of miR-652 on OS cell proliferation was assessed via a CCK-8 assay. The inhibition of miR-652 expression in U2OS and HOS cells resulted in the suppression of cell proliferation at 48 and $72 \mathrm{~h}$ (Fig. $2 \mathrm{~B}$; $\mathrm{P}<0.05)$. In addition, a transwell invasion assay was performed to assess the effect of miR-652 in OS cell invasion. The invasive ability of U2OS and HOS cells was significantly inhibited following treatment with the miR-652 inhibitor (Fig. $2 \mathrm{C}$; $\mathrm{P}<0.05$ ). The results demonstrated that miR-652 functions as an oncogene in OS by regulating cell proliferation and invasion.

KLF9 is a direct target of miR-652 in OS cells. The oncogenic role of miR-652 in OS cells was assessed using bioinformatics analysis to determine potential miR-652 targets. A combination of target sequences were located between the 3'-UTR of KLF9 and miR-652 (Fig. 3A). Subsequently, KLF9 was selected for further experimental identification due to its association with OS formation and progression (23). Luciferase reporter plasmids were constructed and co-transfected with a miR-652 inhibitor or NC inhibitor into U2OS and HOS cells. At $48 \mathrm{~h}$ after transfection, luciferase activity was determined. The results revealed that the downregulation of miR-652 significantly increased the luciferase activity of the plasmid carrying the wild-type 3'-UTR of KLF9 (Fig. 3B; P<0.05). However, the luciferase activity of the plasmid carrying the mutant 3'-UTR was not significantly altered, indicating that miR-652 directly binds to the 3'-UTR of KLF9 (Fig. 3B). In subsequent RT-qPCR and western blot analysis, the expression of KLF9 mRNA (Fig. 3C; P<0.05) and protein (Fig. 3D; $\mathrm{P}<0.05)$ were significantly upregulated in U2OS and HOS cells transfected with the miR-652 inhibitor. To determine whether miR-652 was able to regulate KLF9 expression in hFOB1.19, cells were transfected with miR-652 mimics or an miR-NC. RT-qPCR analysis demonstrated that miR-652 was upregulated in hFOB1.19 cells after transfection with miR-652 mimics (Fig. 3E; P<0.05). KLF9 protein levels were also downregulated in miR-652 mimic-transfected hFOB1.19 cells compared with miR-NC transfected cells (Fig. 3F; P<0.05). The results demonstrated that KLF9 is a direct target gene of miR-652 in OS and normal osteoblast cells.

KLF9 expression is downregulated in OS tissue samples and is inversely correlated with the expression of miR-652. To evaluate the association between miR-652 and KLF9 in OS, KLF9 expression was assessed in 29 pairs of OS and adjacent normal tissue. RT-qPCR analysis revealed that KLF9 mRNA expression was significantly lower in OS tissues compared 

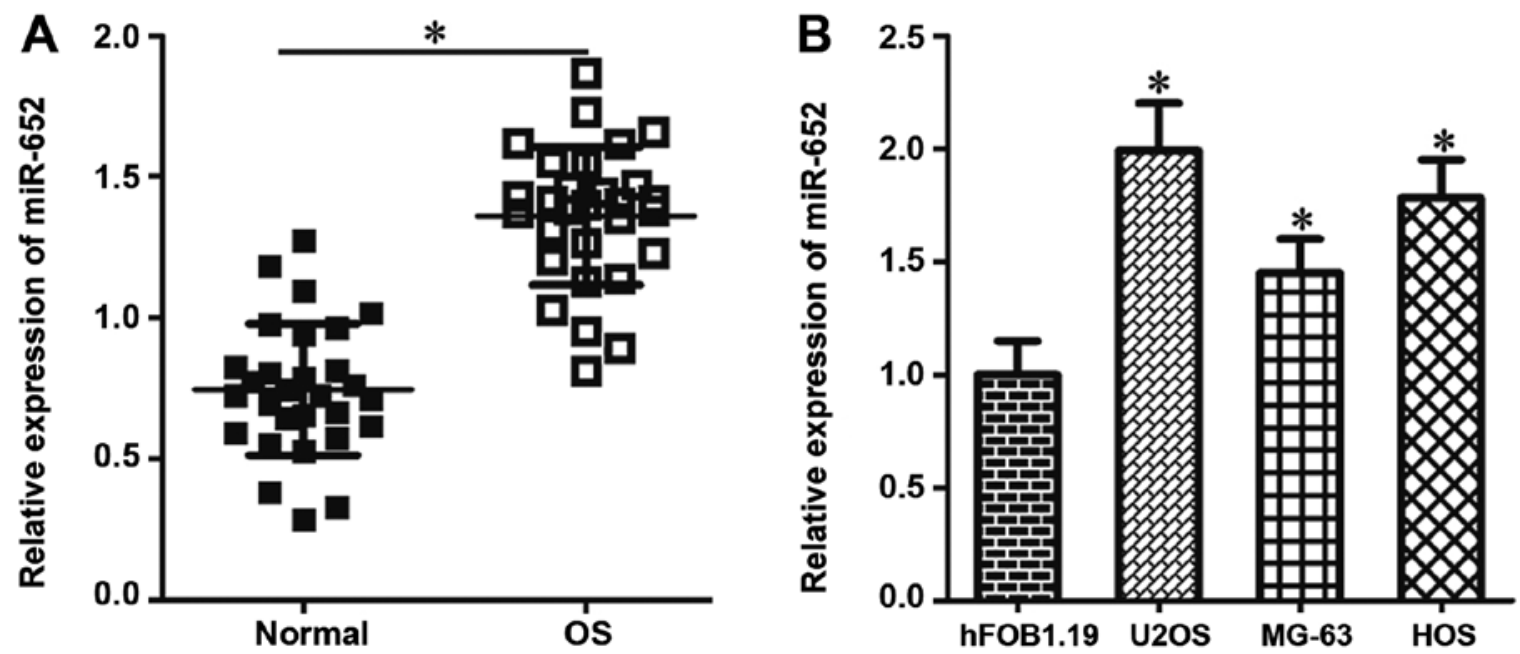

Figure 1. miR-652 upregulation in OS tissues and cell lines. (A) miR-652 expression was detected in 29 pairs of OS and adjacent normal tissues using reverse transcription-quantitative PCR. ${ }^{*} \mathrm{P}<0.05$ vs. adjacent normal tissues. (B) miR-652 expression was determined in three human OS cell lines (U2OS, MG-63 and HOS) and a normal human osteoblast cell line (hFOB1.19). ${ }^{*}$ P<0.05 vs. hFOB1.19. miR, microRNA; OS, osteosarcoma.
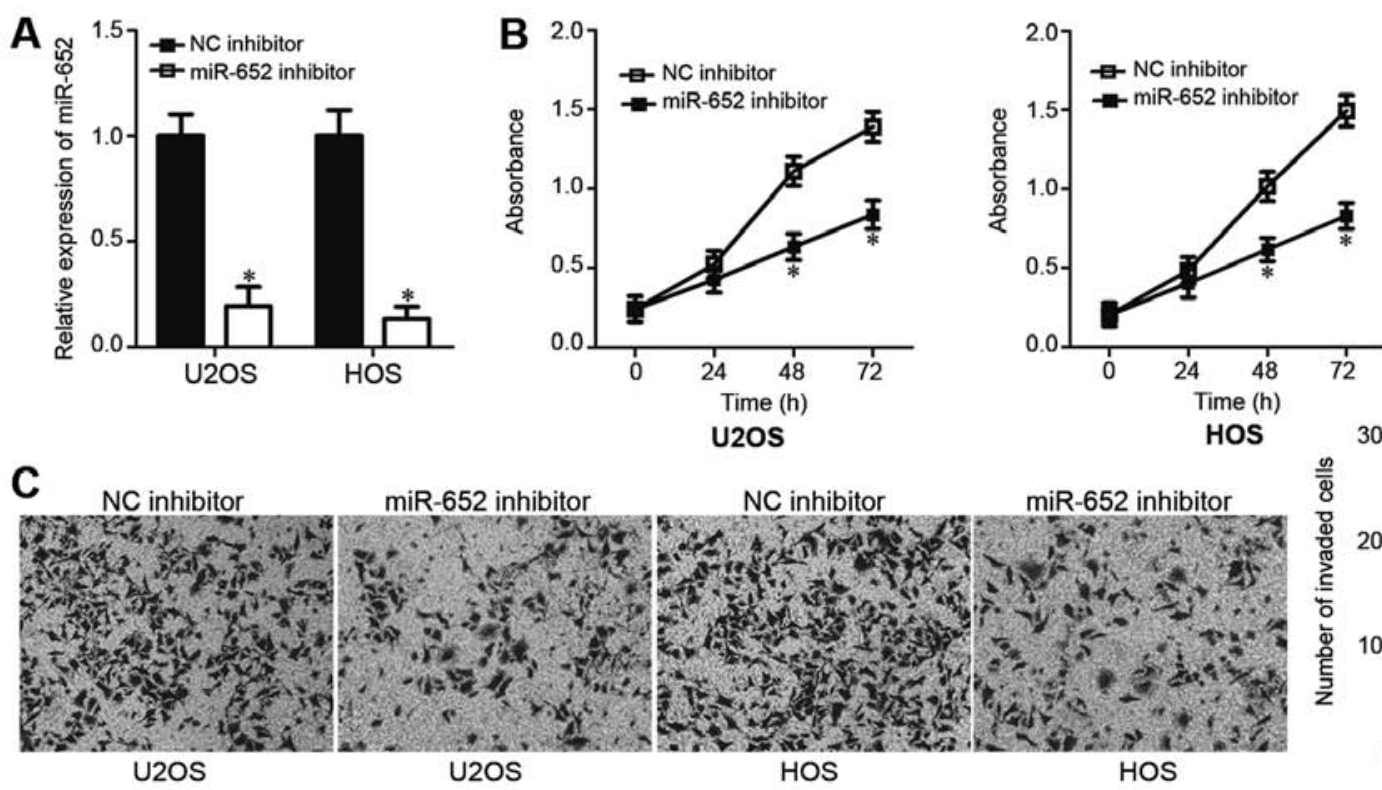
miR-652 inhibitor

Figure 2. miR-652 inhibition inhibits the proliferation and invasion of U2OS and HOS cells. (A) miR-652 expression analysis in U2OS and HOS cells transfected with an miR-652 inhibitor or an NC inhibitor. " $\mathrm{P}<0.05$ vs. NC inhibitor. (B) Cell counting kit-8 assay analysis of miR-652 inhibitor or NC inhibitor-transfected U2OS and HOS cell proliferation. ${ }^{*} \mathrm{P}<0.05$ vs. NC inhibitor. (C) Transwell invasion assay analysis of the invasive ability of U2OS and HOS cells following treatment with a miR-652 inhibitor or an NC inhibitor (magnification x200). "P<0.05 vs. the NC inhibitor. miR, microRNA; NC, negative control.

with adjacent normal tissues (Fig. 4A; $\mathrm{P}<0.05$ ). Furthermore, Spearman's correlation analysis identified an inverse correlation between miR-652 and KLF9 mRNA in OS tissues (Fig. 4B; $r=-0.5510 ; \mathrm{P}=0.0019)$. The results indicated that the downregulation of KLF9 in OS tissue is at least, in part, induced by the overexpression of miR- 652 .

KLF9 knockdown counteracts the miR-652 inhibitor-induced suppression of $O S$ cell proliferation and invasion. As aforementioned, miR-652 was implicated in the regulation of OS cell proliferation and invasion and KLF9 was validated as a direct target of miR-652. Therefore, whether KLF9 mediated the effects of miR-652 was subsequently assessed.
siRNA-mediated knockdown was confirmed in U2OS and Hos cells (Fig. 5A) The siRNA-mediated knockdown of KLF9 was performed in miR-652 inhibitor-transfected U2OS and HOS cells. Western blot analysis demonstrated that the upregulation of KLF9 protein expression in U2OS and HOS cells induced by the miR-652 inhibitor was recovered by co-transfection with KLF9 siRNA (Fig. 5B; P<0.05). CCK-8 and transwell invasion assays indicated that recovered KLF9 expression counteracted the impaired proliferation of U2OS and HOS cells (Fig. 5C; $\mathrm{P}<0.05$ ) and invasion (Fig. 5D; $\mathrm{P}<0.05$ ) induced by the miR- 652 inhibitor at 48 and $72 \mathrm{~h}$. The results indicated that KLF9 mediated the functional roles of miR-652 in OS cell proliferation and invasion. 
A

KLF9 3'-UTR wild-type 5' ...GAUUAAAAAAAAAAACGCCAUAG... 3' hsa-miR-652 3, GUGUUGGGAUCACCGCGGUAA KLF9 3'-UTR mutant 5 '...GAUUAAAAAAAAAAAGCGGUAG... 3',
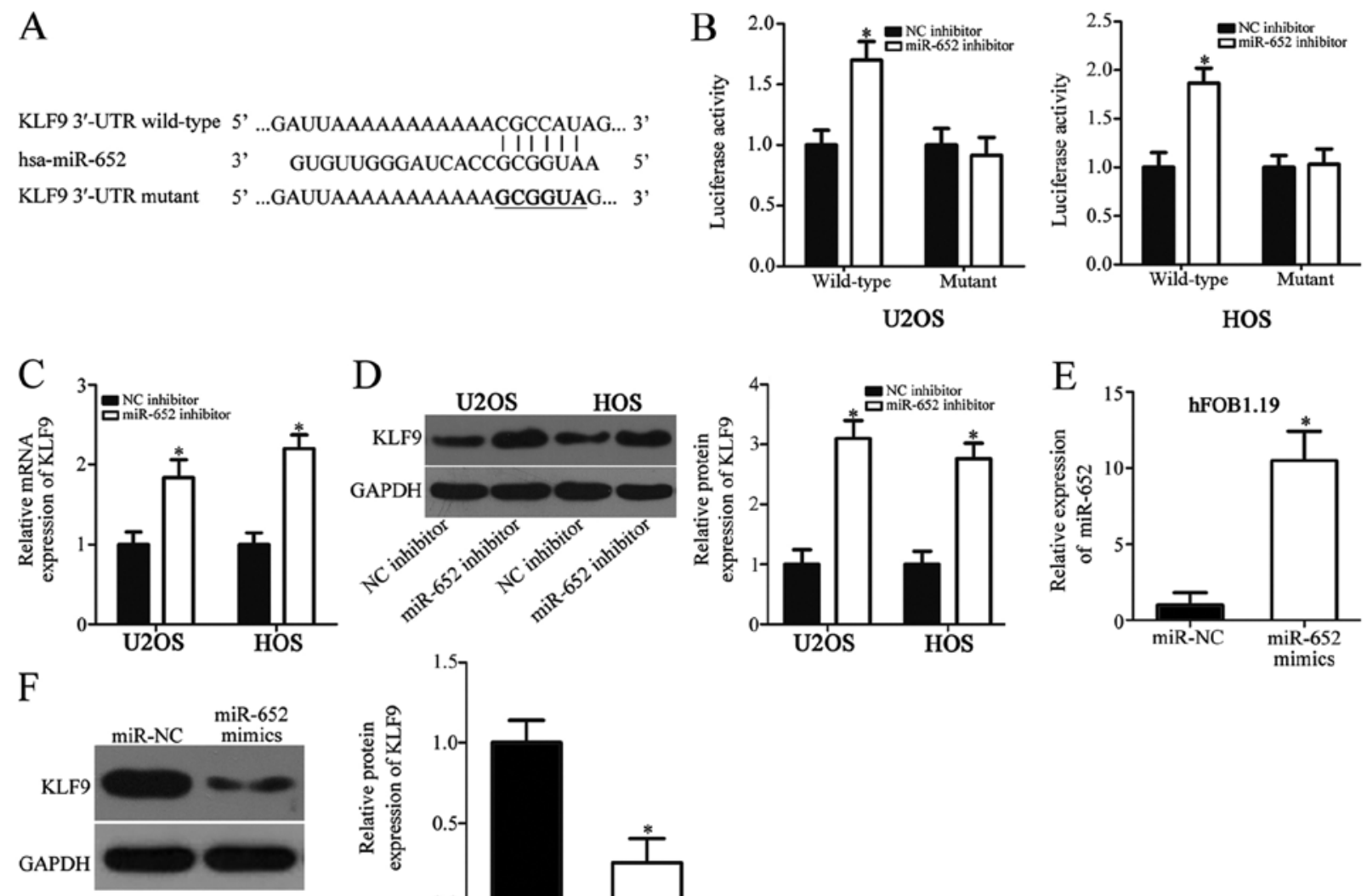

hFOB1.19
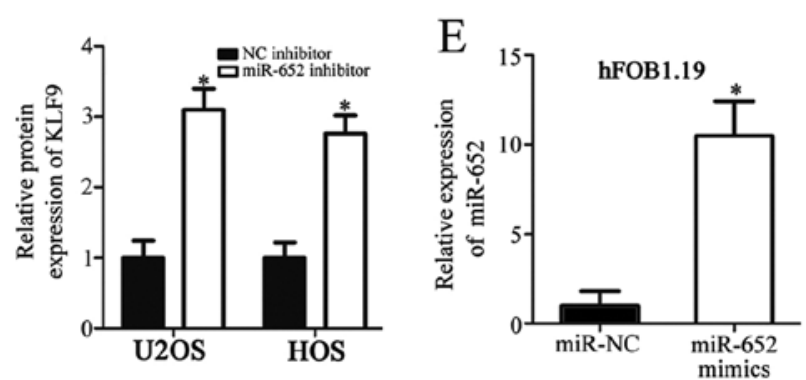

Figure 3. KLF9 is a direct target of miR-652 in osteosarcoma cells. (A) Wild-type and mutant miR-652 binding sequences in the 3'-UTR of KLF9. (B) Luciferase reporter assay analysis of U2OS and HOS cells transfected with the pGL3-KLF9-3'-UTR wild-type or pGL3-KLF9-3'-UTR mutant and miR-652 inhibitor or NC inhibitor. "P<0.05 vs. NC inhibitor. RT-qPCR and western blot analysis of KLF9 (C) mRNA and (D) protein levels in U2OS and HOS cells following treatment with miR-652 inhibitor or NC inhibitor. ${ }^{\mathrm{P}}<0.05$ vs. NC inhibitor. (E) RT-qPCR analysis of miR-652 expression in hFOB1.19 cells after transfection with miR-652 mimics. * $\mathrm{P}<0.05$ vs. miR-NC. (F) Western blot analysis of KLF9 protein expression in miR-652 mimics or miR-NC-transfected hFOB1.19 cells. ${ }^{*} \mathrm{P}<0.05$ vs. miR-NC. KLF9, kruppel-like factor 9; miR, microRNA; UTR, untranslated region; NC, negative control; RT-q, reverse transcription-quantitative.
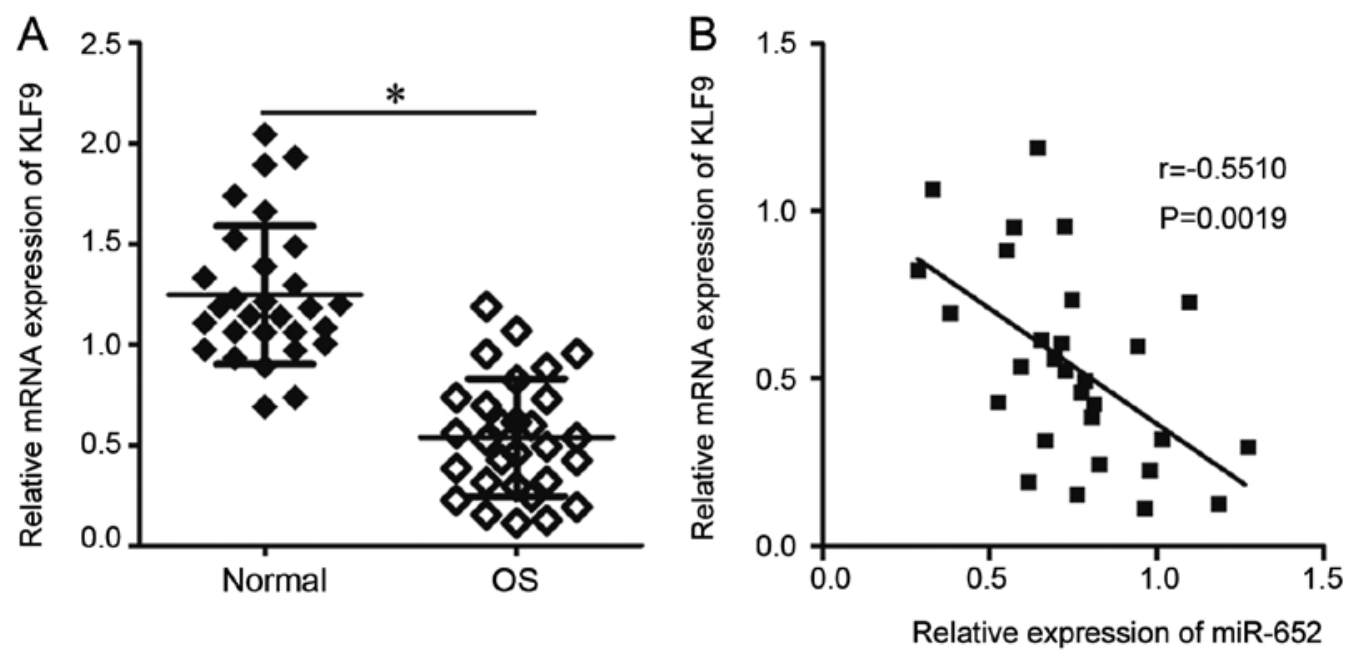

Figure 4. miR-652 and KLF9 expression in OS tissues. (A) Reverse transcription-quantitative PCR analysis of KLF9 mRNA expression in OS and adjacent normal tissues. " $\mathrm{P}<0.05$ vs. adjacent normal tissues. (B) Spearman's correlation analysis of the association between miR-652 and KLF9 mRNA expression in OS tissues ( $\mathrm{r}=-0.5510 ; \mathrm{P}=0.0019)$. miR, microRNA; KLF9, kruppel-like factor 9; OS, osteosarcoma.

\section{Discussion}

Previous studies have determined that a variety of miRNAs are abnormally expressed in OS and that their abnormal expression serves a key role in malignant development $(15,16,24)$. Understanding the mechanism responsible for OS carcinogenesis and progression is therefore critical for the development of effective therapeutic strategies for this 
A
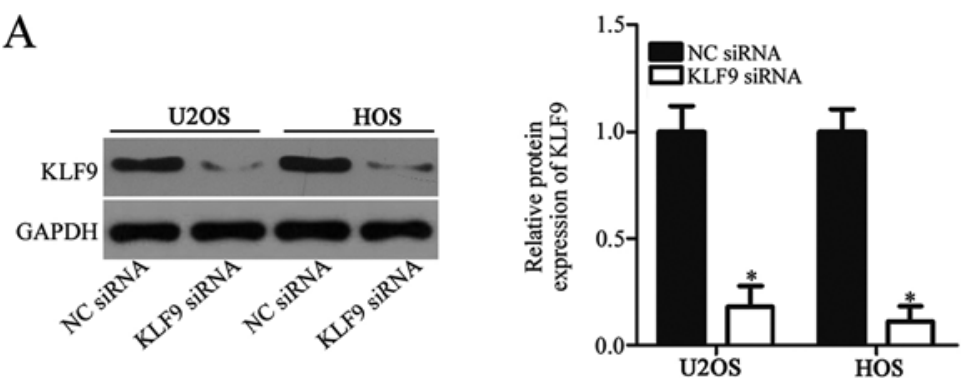

B
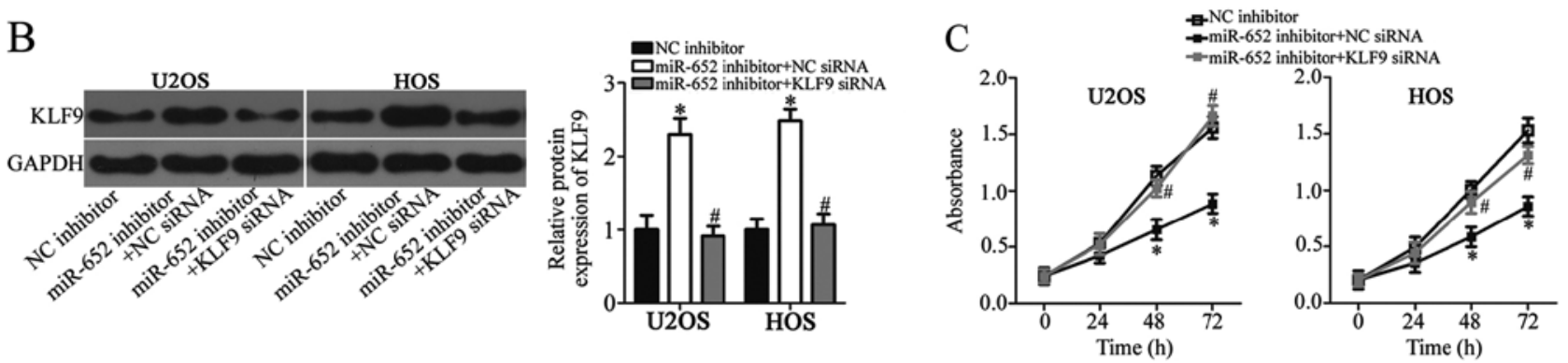

$\mathrm{D}$ U2OS

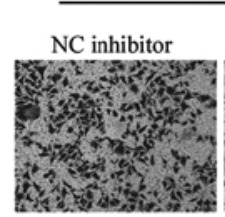
miR-652 inhibitor miR-652 inhibitor $+\mathrm{NC}$ siRNA
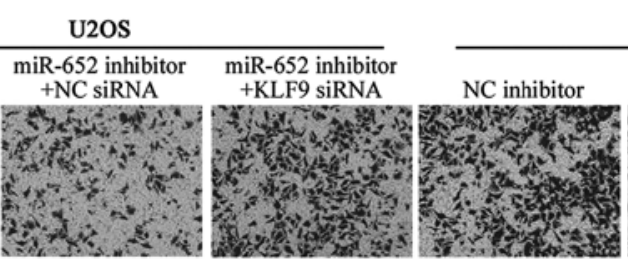

HOS

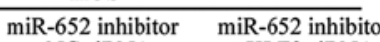
miR-652 inhibito
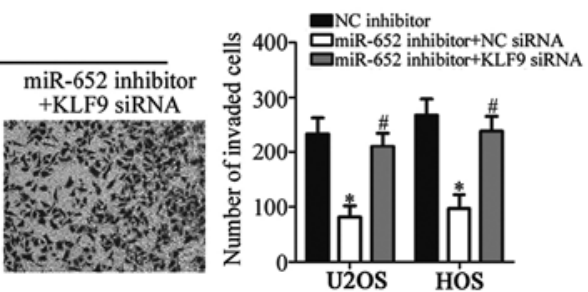

Figure 5. Role of KLF9 upregulation in miR-652 inhibitor-induced suppression of U2OS and HOS cell proliferation and invasion. (A) Western blot analysis of KLF9 expression in U2OS and HOS cells transfected with KLF9 siRNA or NC siRNA. "P<0.05 vs. NC siRNA. (B) Western blot analysis of KLF9 protein expression in U2OS and HOS cells transfected with miR-652 inhibitor, followed by co-transfection with KLF9 siRNA or NC siRNA."P $<0.05$ vs. NC inhibitor; ${ }^{\#} \mathrm{P}<0.05$ vs. miR-652 inhibitor + NC siRNA. (C) Cell counting kit-8 assay analysis of the proliferative ability of the aforementioned cells. ${ }^{*} \mathrm{P}<0.05$ vs. NC inhibitor; ${ }^{~} \mathrm{P}<0.05$ vs. miR-652 inhibitor $+\mathrm{NC}$ siRNA. (D) Transwell invasion assays analysis of the effects of U2OS and HOS treated cells on cell invasion (magnification $\mathrm{x} 200$ ). ${ }^{\text {"P }}<0.05$ vs. NC inhibitor; ${ }^{\prime} \mathrm{P}<0.05$ vs. miR-652 inhibitor + NC siRNA. miR, microRNA; KLF9, kruppel-like factor 9; siRNA, small interfering RNA; NC, negative control.

disease (25). An in-depth understanding of the functional roles and underlying mechanisms of dysregulated miRNAs in OS may be significant for cancer research and important for the identification of novel therapeutic targets. To the best of our knowledge, the current study assessed the expression status of miR-652 in OS and determined the detailed roles of miR-652 in OS progression for the first time. The molecular mechanisms of miR-652 activity in OS cells were also assessed.

miR-652 has been revealed to be downregulated in pancreatic cancer tissues and cell lines. A decreased expression of miR-652 was significantly correlated with tumor stage, lymphatic invasion and metastasis in patients with pancreatic cancer (20). In contrast, miR-652 is highly expressed in non-small cell lung cancer tissue and cell lines. The upregulation of miR-652 was associated with lymph node metastasis, TNM stage and prognosis in patients with non-small cell lung cancer (21). These inconsistent results indicate that the expression of miR-652 exhibits tissue specificity in human malignancies. However, the expression status of miR-652 in OS remains unclear. In the current study, RT-qPCR was performed to detect miR-652 and the results revealed that it was significantly upregulated in OS tissues and cell lines. The results also demonstrated that miR-652 may be used as a potential biomarker in the diagnosis of OS.
The dysregulation of miR- 652 contributes to the malignant phenotype of human cancer. miR-652 has been identified as a tumor suppressor in pancreatic cancer (20). The upregulation of miR-652 expression abolishes the acidity-induced epithelial-mesenchymal transition of pancreatic cancer cells via the negative regulation of zinc finger E-Box binding homeobox 1 (20). miR-652 also serves oncogenic roles in the progression of non-small cell lung cancer and directly targets lethal giant larvae 1 to affect cell proliferation, apoptosis, migration and invasion (21). However, the roles of miR-652 in OS progression are unknown. In the current study, CCK-8 and transwell invasion assays demonstrated that miR-652 inhibition resulted in a significant suppression of OS cell proliferation and invasion. The results indicated that miR-652 may be a promising therapeutic target for anticancer therapy.

The identification of the direct target genes of miR-652 is important for understanding its role in carcinogenesis and progression (26). The results of the present study demonstrated that KLF9 was a direct target gene of miR-652 in OS cells. Bioinformatic predictions indicated KLF9 as a putative target of miR-652. Subsequently, a luciferase reporter assay, RT-qPCR and western blot analysis revealed that miR-652 regulated the expression of KLF9 by binding to its 3'-UTR in OS cells. KLF9 was also downregulated in OS tissues and this downregulation was inversely correlated with miR-652 
expression. Furthermore, the downregulation of KLF9 decreased the miR-652 inhibitor-induced suppression of OS cell proliferation and invasion. These results may provide sufficient evidence to support the hypothesis that KLF9 is a direct target gene of miR-652 in OS cells.

KLF9 is a member of the KLF family (27) and has been previously reported to be downregulated in various types of human malignancy, including pancreatic ductal adenocarcinoma (28), hepatocellular carcinoma (29), colorectal cancer (30) and prostate cancer (31). KLF9 serves as a tumour suppressor at tumour onset and development, affecting cell proliferation, apoptosis, metastasis and tumorigenicity (32-34). Small quantities of KLF9 are expressed in OS and this low expression level may modulate OS aggression (23). The present study is, to the best of our knowledge, the first to demonstrate that miR-652 regulated KLF9 expression and may therefore inhibit the progression of OS. The results of the current study revealed that miR-652 inhibition or KLF9 restoration may be an effective therapeutic technique to treat patients with OS in the future.

In the current study, the correlation between miR-652 expression and clinicopathological factors in patients with OS was not determined. Therefore, in following investigations, the collection of more tissues and the determination of clinical significance should be assessed. The influence of miR-652 overexpression in the malignant development of OS cells was also not clarified. miR-652 mimics should be utilized in future studies to increase endogenous miR-652 expression. In addition, a series of functional experiments should be performed to evaluate the effects of miR-652 overexpression in OS cells. The regulatory effects of miR-652 in the apoptosis and migration of OS cells was not assessed in the present study, and as such, flow cytometry analysis and transwell migration assay should be applied in future studies to determine this.

In conclusion, miR-652 was upregulated in OS tissues and cell lines. The downregulation of miR-652 also inhibited the proliferation and invasion of OS cells by targeting KLF9 directly. All the results obtained in the current study may provide a basis for the identification of novel therapeutic targets for the prevention of OS and for OS therapy.

\section{Acknowledgements}

Not applicable.

\section{Funding}

No funding was received.

\section{Availability of data and materials}

The datasets used and/or analyzed during the current study are available from the corresponding author on reasonable request.

\section{Authors' contributions}

XL designed the current study, wrote the manuscript and performed the luciferase reporter assay. YJ and LY performed reverse transcription-quantitative PCR, the cell counting kit- 8 assay, the transwell invasion assay and western blot analysis. All authors have read and approved the final draft.

\section{Ethics approval and consent to participate}

The present study was approved by the Ethics Committee of Henan University and was performed in accordance with the Declaration of Helsinki and the guidelines of the Ethics Committee of The First Affiliated Hospital of Henan University. Written informed consent was provided by all participations prior to their enrollment.

\section{Patient consent for publication}

Not applicable.

\section{Competing interests}

The authors declare that they have no competing interests.

\section{References}

1. Ando K, Mori K, Verrecchia F, Marc B, Redini F and Heymann D: Molecular alterations associated with osteosarcoma development. Sarcoma 2012: 523432, 2012.

2. Picci P: Osteosarcoma (osteogenic sarcoma). Orphanet J Rare Dis 2: 6, 2007.

3. Tan ML, Choong PF and Dass CR: Osteosarcoma: Conventional treatment vs. Gene therapy. Cancer Biol Ther 8: 106-117, 2009.

4. Ferrari S, Palmerini E, Staals EL, Mercuri M, Franco B, Picci P and Bacci G: The treatment of nonmetastatic high grade osteosarcoma of the extremity: Review of the italian rizzoli experience. Impact on the future. Cancer Treat Res 152: 275-287, 2009.

5. Pan Y, Lu L, Chen J, Zhong Y and Dai Z: Identification of potential crucial genes and construction of MicroRNA-MRNA negative regulatory networks in osteosarcoma. Hereditas 155: 21, 2018.

6. To KK, Tong CW, Wu M and Cho WC: MicroRNAs in the prognosis and therapy of colorectal cancer: From bench to bedside. World J Gastroenterol 24: 2949-2973, 2018.

7. Yuan HL, Wang T and Zhang KH: MicroRNAs as potential biomarkers for diagnosis, therapy and prognosis of gastric cancer. Onco Targets Ther 11: 3891-3900, 2018.

8. Sharma $N$ and Baruah MM: The microRNA signatures: Aberrantly expressed miRNAs in prostate cancer. Clin Transl Oncol 21: 126-144, 2018.

9. Han K, Chen X, Bian N, Ma B, Yang T, Cai C, Fan Q, Zhou Y and Zhao TB: MicroRNA profiling identifies MiR-195 suppresses osteosarcoma cell metastasis by targeting CCND1. Oncotarget 6: 8875-8889, 2015

10. Bartel DP: MicroRNAs: Genomics, biogenesis, mechanism, and function. Cell 116: 281-297, 2004.

11. Chen X, Jia C, Jia C, Jin X and Gu X: MicroRNA-374a Inhibits aggressive tumor biological behavior in bladder carcinoma by suppressing wnt/ $\beta$-Catenin signaling. Cell Physiol Biochem 48: 815-826, 2018

12. Tao Y, Ma C, Fan Q, Wang Y, Han T and Sun C: MicroRNA-1296 facilitates proliferation, migration and invasion of colorectal cancer cells by targeting SFPQ. J Cancer 9: 2317-2326, 2018.

13. Yang JZ, Bian L, Hou JG and Wang HY: MiR-550a-3p promotes non-small cell lung cancer cell proliferation and metastasis through down-regulating TIMP2. Eur Rev Med Pharmacol Sci 22: 4156-4165, 2018.

14. Wu Y, Huang J, Xu H and Gong Z: Over-Expression of MiR-15a-3p enhances the radiosensitivity of cervical cancer by targeting tumor protein D52. Biomed Pharmacother 105: 1325-1334, 2018.

15. Leichter AL, Sullivan MJ, Eccles MR and Chatterjee A: MicroRNA expression pat terns and signalling pathways in the development and progression of childhood solid tumours. Mol Cancer 16: 15, 2017.

16. Kim YH, Goh TS, Lee CS, Oh SO, Kim JI, Jeung SH and Pak K: Prognostic value of microRNAs in osteosarcoma: A metaanalysis. Oncotarget 8: 8726-8737, 2017. 
17. Wang Z, Zheng C, Jiang K, He J, Cao X and Wu S: MicroRNA-503 suppresses cell proliferation and invasion in osteosarcoma via targeting insulin-like growth factor 1 receptor. Exp Ther Med 14: $1547-1553,2017$.

18. Ma C, Han J, Dong D and Wang N: MicroRNA-152 suppresses human osteosarcoma cell proliferation and invasion by targeting E2F transcription factor 3. Oncol Res 26: 765-773, 2018.

19. Liu K, Sun X, Zhang Y, Liu L and Yuan Q: MiR-598: A tumor suppressor with biomarker significance in osteosarcoma. Life Sci 188: 141-148, 2017.

20. Deng S, Li X, Niu Y, Zhu S, Jin Y, Deng S, Chen J, Liu Y, He C, Yin T, et al: MiR-652 inhibits acidic microenvironment-induced epithelial-mesenchymal transition of pancreatic cancer cells by targeting ZEB1. Oncotarget 6: 39661-39675, 2015.

21. Yang W, Zhou C, Luo M, Shi X, Li Y, Sun Z, Zhou F, Chen Z and He J: MiR-652-3p is upregulated in non-small cell lung cancer and promotes proliferation and metastasis by directly targeting Lgl1. Oncotarget 7: 16703-16715, 2016.

22. Livak KJ and Schmittgen TD: Analysis of relative gene expression data using real-time quantitative PCR and the 2(-Delta Delta C(T)) method. Methods 25: 402-408, 2001.

23. Peng N, Miao Z, Wang L, Liu B, Wang G and Guo X: MiR-378 promotes the cell proliferation of osteosarcoma through down-regulating the expression of kruppel-like factor 9. Biochem Cell Biol 96: 515-521, 2018.

24. Sampson VB, Yoo S, Kumar A, Vetter NS and Kolb EA: MicroRNAs and potential targets in osteosarcoma: Review. Front Pediatr 3: 69, 2015.

25. Smolle MA, Leithner A, Posch F, Szkandera J, Liegl-Atzwanger B and Pichler M: MicroRNAs in different histologies of soft tissue sarcoma: A comprehensive review. Int J Mol Sci 18: E1960, 2017.
26. Yao J, Zhang P, Li J and Xu W: MicroRNA-215 acts as a tumor suppressor in breast cancer by targeting AKT serine/threonine kinase 1. Oncol Lett 14: 1097-1104, 2017.

27. McConnell BB and Yang VW: Mammalian kruppel-like factors in health and diseases. Physiol Rev 90: 1337-1381, 2010.

28. Mao Z, Fan X, Zhang J, Wang X, Ma X, Michalski CW and Zhang Y: KLF9 is a prognostic indicator in human pancreatic ductal adenocarcinoma. Anticancer Res 37: 3795-3799, 2017.

29. Sun J, Wang B, Liu Y, Zhang L, Ma A, Yang Z, Ji Y and Liu Y: Transcription factor KLF9 suppresses the growth of hepatocellular carcinoma cells vivo and positively regulates p53 expression. Cancer Lett 355: 25-33, 2014.

30. Kang L, $\mathrm{Lu} \mathrm{B}, \mathrm{Xu} \mathrm{J}, \mathrm{Hu} \mathrm{H}$ and Lai M: Downregulation of kruppel-like factor 9 in human colorectal cancer. Pathol Int 58: 334-338, 2008.

31. Shen P, Sun J, Xu G, Zhang L, Yang Z, Xia S, Wang Y, Liu Y and Shi G: KLF9, a transcription factor induced in flutamide-caused cell apoptosis, inhibits AKT activation and suppresses tumor growth of prostate cancer cells. Prostate 74: 946-958, 2014

32. Bai XY, Li S, Wang M, Li X, Yang Y, Xu Z, Li B, Li Y, Xia K, Chen $\mathrm{H}$ and $\mathrm{Wu} \mathrm{H}$ : Kruppel-like factor 9 down-regulates matrix metalloproteinase 9 transcription and suppresses human breast cancer invasion. Cancer Lett 412: 224-235, 2018.

33. Huang S, Wang C, Yi Y, Sun X, Luo M, Zhou Z, Li J, Cai Y, Jiang $\mathrm{X}$ and $\mathrm{Ke} \mathrm{Y}$ : Kruppel-like factor 9 inhibits glioma cell proliferation and tumorigenicity via downregulation of miR-21. Cancer Lett 356: 547-555, 2015.

34. Zhang QH,DouHT, Tang YJ,Su S and Liu PS: Lentivirus-mediated knockdown of Kruppel-like factor 9 inhibits the growth of ovarian cancer. Arch Gynecol Obstet 291: 377-382, 2015. 